\title{
Dynamics of Synaptic Transmission between Fast-Spiking Interneurons and Striatal Projection Neurons of the Direct and Indirect Pathways
}

\author{
Henrike Planert, ${ }^{1,3}$ Susanne N. Szydlowski, ${ }^{1,3}$ J. J. Johannes Hjorth, ${ }^{2,3}$ Sten Grillner, ${ }^{1,3 *}$ and Gilad Silberberg ${ }^{1,3 *}$ \\ ${ }^{1}$ Department of Neuroscience, Karolinska Institute, 17177 Stockholm, Sweden, ${ }^{2}$ Computational Biology, Royal Institute of Technology, 10691 Stockholm, \\ Sweden, and ${ }^{3}$ Stockholm Brain Institute, Karolinska Institutet, 17177 Stockholm, Sweden
}

The intrastriatal microcircuit is a predominantly inhibitory GABAergic network comprised of a majority of projection neurons [medium spiny neurons (MSNs)] and a minority of interneurons. The connectivity within this microcircuit is divided into two main categories: lateral connectivity between MSNs, and inhibition mediated by interneurons, in particular fast spiking (FS) cells. To understand the operation of striatum, it is essential to have a good description of the dynamic properties of these respective pathways and how they affect different types of striatal projection neurons.

We recorded from neuronal pairs, triplets, and quadruplets in slices of rat and mouse striatum and analyzed the dynamics of synaptic transmission between MSNs and FS cells. Retrograde fluorescent labeling and transgenic EGFP (enhanced green fluorescent protein) mice were used to distinguish between MSNs of the direct (striatonigral) and indirect (striatopallidal) pathways. Presynaptic neurons were stimulated with trains of action potentials, and activity-dependent depression and facilitation of synaptic efficacy was recorded from postsynaptic neurons. We found that FS cells provide a strong and homogeneously depressing inhibition of both striatonigral and striatopallidal MSN types. Moreover, individual FS cells are connected to MSNs of both types. In contrast, both MSN types receive sparse and variable, depressing and facilitating synaptic transmission from nearby MSNs. The connection probability was higher for pairs with presynaptic striatopallidal MSNs; however, the variability in synaptic dynamics did not depend on the types of interconnected MSNs. The differences between the two inhibitory pathways were clear in both species and at different developmental stages. Our findings show that the two intrastriatal inhibitory pathways have fundamentally different dynamic properties that are, however, similarly applied to both direct and indirect striatal projections.

\section{Introduction}

The striatum plays a major role in motor learning and in determining the pattern of behavior that is selected at any given time in all vertebrates (Graybiel et al., 1994; Barnes et al., 2005; Grillner et al., 2005; McHaffie et al., 2005). It receives converging glutamatergic input from cortex and thalamus, dopaminergic projections from substantia nigra pars compacta, as well as selective GABAergic input from the external pallidum (Bevan et al., 1998), all of which interact with the primarily GABAergic intrinsic striatal microcircuitry.

The striatal microcircuit consists mostly of GABAergic neurons, of which medium spiny neurons (MSNs), the projection neurons, constitute the vast majority (Wilson and Groves, 1980;

\footnotetext{
Received 0ct. 15, 2009; revised Dec. 17, 2009; accepted Jan. 18, 2010.

This work was supported by the European Commission Coordination Action Network of European Neuroscience Institutes Contract LSHM-CT-2005-19063, the Human Frontier Science Program, the Swedish Science Research Council (VR-M), the Swedish Parkinson's Disease Foundation (Parkinsonfonden), and the European Union Seventh Framework Programme (Cortex and Select and Act). We thank Brita Robertson and Thomas Berger for help with the morphological staining and retrograde labeling.

*S.G. and G.S. contributed equally to this work.

Correspondence should be addressed to either Sten Grillner or Gilad Silberberg at the above address. E-mail: sten.grillner@ki.se or gilad.silberberg@ki.se.

DOI:10.1523/JNEUROSCI.5139-09.2010

Copyright $\odot 2010$ the authors $\quad 0270-6474 / 10 / 303499-09 \$ 15.00 / 0$
}

Graveland and DiFiglia, 1985). Synaptic connectivity between MSNs has long been predicted from their morphology (Wilson and Groves, 1980) and in recent years was confirmed at the electrophysiological level (Czubayko and Plenz, 2002; Tunstall et al., 2002; Koós et al., 2004; Venance et al., 2004; Tecuapetla et al., 2007, 2009; Taverna et al., 2008). Synaptic connectivity between MSNs is sparse (Czubayko and Plenz, 2002; Tunstall et al., 2002; Koós et al., 2004; Venance et al., 2004; Taverna et al., 2008) and formed mainly on dendritic shafts and spines (Wilson and Groves, 1980; Somogyi et al., 1981). MSNs also receive strong GABAergic input from the different types of interneurons, with input from fast spiking (FS) cells mainly targeted to the soma and perisomatic regions (Kita et al., 1990; Bennett and Bolam, 1994; Koós and Tepper, 1999; Taverna et al., 2007).

The connectivity between MSNs of the direct (striatonigral) and indirect (striatopallidal) pathways was recently reported (Taverna et al., 2008), showing a higher prevalence of connections formed by striatopallidal neurons onto target MSNs than those formed by striatonigral MSNs. MSNs of the two pathways also differ in the long-term synaptic plasticity of glutamatergic inputs and their dopaminergic modulation (Surmeier et al., 2007; Day et al., 2008). However, the short-term plasticity of afferent excitatory inputs does not differ significantly between direct- and 
indirect-pathway MSNs (Ding et al., 2008). In the striatum, GABAergic connectivity between MSNs is assumed to have a different functional role than the GABAergic synapses from interneurons onto MSNs (Koós and Tepper, 1999; Koós et al., 2004; Gustafson et al., 2006; Tecuapetla et al., 2007), with the latter suggested to mediate feedforward inhibition after cortical activation (Mallet et al., 2005). To understand the function of these respective intrastriatal synaptic pathways, it is necessary to determine their dynamic properties and how they differ across cell types. In this study, we analyzed the dynamic properties of synapses between identified neuronal pairs in the rodent striatum, in particular the lateral connections between striatonigral and striatopallidal MSNs, and the synaptic connections they receive from FS interneurons. These results have been partly reported in abstract form (Planert et al., 2008).

\section{Materials and Methods}

Slice preparation and recordings. All experiments were performed according to the guidelines of the Stockholm municipal committee for animal experiments. Parasagittal and coronal slices were obtained from rats and mice (postnatal days $14-18$ and 21-36, respectively). To distinguish between striatonigral and striatopallidal MSNs, we used transgenic BAC mice expressing enhanced green fluorescent protein (EGFP) in $\mathrm{D}_{1}$ MSNs (Wang et al., 2006b). Slices were cut in ice-cold extracellular solution, kept at $35^{\circ} \mathrm{C}$ for $30 \mathrm{~min}$, and then moved to room temperature before recordings. Whole-cell patch recordings were obtained from striatal neurons at a temperature of $35 \pm 0.5^{\circ} \mathrm{C}$. Neurons were visualized using infrared-differential interference contrast (IR-DIC) microscopy (Zeiss FS Axioskop). Recorded neurons were selected visually and up to four neighboring neurons with lateral somatic distances $<100 \mu \mathrm{m}$ were simultaneously recorded (see Fig. $1 A, B$ ). In experiments in which striatonigral MSNs were labeled, we used a mercury lamp (HBO 100; Zeiss) mounted on the same microscope and a fluorescent filter cube (green or red, depending on the use of GFP or fluorescent beads) to determine the subtype of MSNs, and then switched to IR-DIC to perform whole-cell recordings. Classification of nonlabeled MSNs and interneurons was performed during electrophysiological recordings and, when performed, verified by morphological staining. The extracellular solution (both for cutting and recording) contained the following (in $\mathrm{mm}$ ): $125 \mathrm{NaCl}, 25$ glucose, $25 \mathrm{NaHCO}_{3}, 2.5 \mathrm{KCl}, 2 \mathrm{CaCl}_{2}, 1.25 \mathrm{NaH}_{2} \mathrm{PO}_{4}, 1 \mathrm{MgCl}_{2}$. Recordings were amplified using Axoclamp 2B or MultiClamp 700B amplifiers (Molecular Devices), filtered at $2 \mathrm{kHz}$, digitized $(5-20 \mathrm{kHz})$ using ITC-18 (InstruTECH), and acquired using Igor Pro (Wavemetrics). Patch pipettes were pulled with a Flaming/Brown micropipette puller P-97 (Sutter Instrument) and had an initial resistance of 5-10 M $\Omega$, containing the following (in $\mathrm{mm}$ ): $110 \mathrm{~K}$-gluconate, $10 \mathrm{KCl}, 10 \mathrm{HEPES}, 4 \mathrm{Mg}$-ATP, 0.3 GTP, 10 phosphocreatine, and in a subset of neurons $0.4-0.5 \%$ biocytin. A subset of experiments in retrogradely labeled rat slices was performed with higher internal chloride concentrations, with either $20 \mathrm{~mm}$ K-gluconate and $100 \mathrm{~mm} \mathrm{KCl}$, or $105 \mathrm{~mm} \mathrm{~K}$-gluconate and $30 \mathrm{~mm} \mathrm{KCl}$. The latter concentrations were used for all experiments in mice. Liquid junction potential $(\sim 10 \mathrm{mV})$ was not corrected for in any of the recordings. Recordings were performed both in current- and voltage-clamp mode, with access resistance compensated throughout the experiments. Recordings were discarded when access resistance increased beyond 35 $\mathrm{M} \Omega$.

Retrograde labeling. Striatonigral neurons were labeled by stereotactic injection of fluorescent latex microspheres (Lumafluor) into the substantia nigra pars reticulata of juvenile rats (postnatal day 12). The beads are transported retrogradely by the axons that terminate at the site of injection. The injection procedure has been described in detail previously (Le Bé et al., 2007). In short, rats were anesthetized with an intraperitoneal injection of a mixture of Fentanyl (Fentanyl; B. Braun Melsungen) and medetomidine (Domitor; Orion Pharma), diluted in $0.9 \%$ saline and administered at a final dose of $300 \mu \mathrm{g} / \mathrm{kg}$. The smooth surface of the skull was pierced with a 20 gauge Microlance syringe (BD Biosciences), and red microspheres were injected with a Hamilton syringe at a volume of
$0.4 \mu \mathrm{l}$ over $1 \mathrm{~min}$. Stereotactic coordinates were as follows: $2.2 \mathrm{~mm}$ lateral from midline, $1.1 \mathrm{~mm}$ anterior to lambda, and $6.9 \mathrm{~mm}$ below the skull surface. To allow diffusion of the solution from the injection site, the syringe was left at the place of injection for at least $5 \mathrm{~min}$. Bupivacain (Marcain; $2.5 \mathrm{mg} / \mathrm{ml}$; AstraZeneca) was used as local anesthetic before the wound was closed with chirurgical glue (Histoacryl; Aesculap). The analgesic karprofen (Rimadyl; Pfizer) was administered subcutaneously at $5 \mathrm{mg} / \mathrm{kg}$, and the rats were awakened with intraperitoneal injections of a mixture of atipamezole (Antisedan; Orion Pharma; $1 \mathrm{mg} / \mathrm{kg})$ and naloxone $(0.1 \mathrm{mg} / \mathrm{kg})$, diluted in $0.9 \%$ saline. After surgery, the pups were returned to their mother's cage. Slices were visualized under fluorescent microscopy, and only slices in which the striatum was clearly labeled were used for electrophysiological recordings.

Stimulation protocols and analysis. Recorded neurons were subject to various stimulation protocols to determine the synaptic and intrinsic electrical properties. Synaptic connections were identified and characterized by stimulation of a presynaptic cell with a train $(10,20,40$, or $70 \mathrm{~Hz})$ of eight strong and brief current pulses (0.5-2 nA; $3 \mathrm{~ms}$ ), followed by a so-called recovery test pulse between 500 and $600 \mathrm{~ms}$ after the train, all reliably eliciting action potentials (APs). Postsynaptic neurons were held near $-80 \mathrm{mV}$ to ensure depolarizing responses to GABAergic synapses. For analysis of synaptic properties, the average postsynaptic trace of $>20$ sweeps was examined for the existence of synaptic responses. Synaptic responses were obtained in current-clamp and voltage-clamp modes, and in both cases exhibited similar dynamic properties.

Parameters describing the dynamics of recorded synapses included the synaptic utilization parameter $(U)$ (equivalent to the average release probability) and the time constants of recovery from depression $(D)$ and facilitation $(F)$. These parameters were extracted using the model for synaptic dynamics as previously described (Markram et al., 1998; Tsodyks et al., 1998). Amplitudes of postsynaptic responses are calculated from postsynaptic responses to presynaptic depolarizing pulses. To extract correct amplitudes of postsynaptic responses lying on the decay phase of previous responses, the synaptic decay was fitted by an exponential curve and subtracted. The amplitude of the postsynaptic response, $\mathrm{PSP}_{n}$, is a product of the fraction of available resources, $R_{n}$, and a facilitating utilization factor, $u_{n}$, scaled by the absolute synaptic efficacy, $A_{\text {se }}$, as follows:

$$
\mathrm{PSP}_{n}=A_{\mathrm{se}} R_{n} u_{n}
$$

The utilization factor is increased by each AP and decays back toward $U$ in the time between APs, $t_{\text {ISI }}$. This process is described by utilization of the synaptic efficiency in the first $\operatorname{AP}\left(u_{0}=U\right)$, and the recovery time constant from facilitation, $F$, as follows:

$$
u_{n+1}=u_{n} \exp \left(-\frac{t_{\mathrm{ISI}}}{F}\right)+U\left(1-u_{n} \exp \left(-\frac{t_{\mathrm{ISI}}}{F}\right)\right)
$$

Each AP uses the fraction $u_{n}$ from the synaptic resources, $R_{n}$, which then recovers to a value of 1 at rate of $D$ as follows:

$$
R_{n+1}=R_{n}\left(1-u_{n}\right) \exp \left(\frac{-t_{\mathrm{ISI}}}{D}\right)+1-\exp \left(\frac{-t_{\mathrm{ISI}}}{D}\right) .
$$

The model parameters describing each of the analyzed connections were extracted from the average synaptic responses.

Morphological staining. Slices in which recorded cells were biocytin filled were fixed in $0.1 \mathrm{M}$ phosphate buffer $(\mathrm{PB})$ containing $4 \%$ paraformaldehyde overnight before the staining procedure. Slices stained for light microscopy were first incubated with $0.6 \% \mathrm{H}_{2} \mathrm{O}_{2}$ in methanol, rinsed in $0.01 \mathrm{M}$ PBS, and thereafter incubated with the $\mathrm{ABC}$ elite kit (Vector Laboratories) for $3 \mathrm{~h}$. The ABC complex was diluted with $0.3 \%$ Triton X-100 in $0.1 \mathrm{M}$ PB. After rinsing with $0.01 \mathrm{M}$ PBS, the slices were incubated with DAB (The ImmPACT DAB kit; Vector Laboratories) for 4-10 min. Slices were then mounted on gelatin-coated slides, dehydrated, cleared in xylene, and coverslipped with DPX (dibutylphthalate polystyrene xylene) (Sigma-Aldrich). Slices used for fluorescent microscopy were incubated overnight with streptavidin-Cy2 (1:1000; 

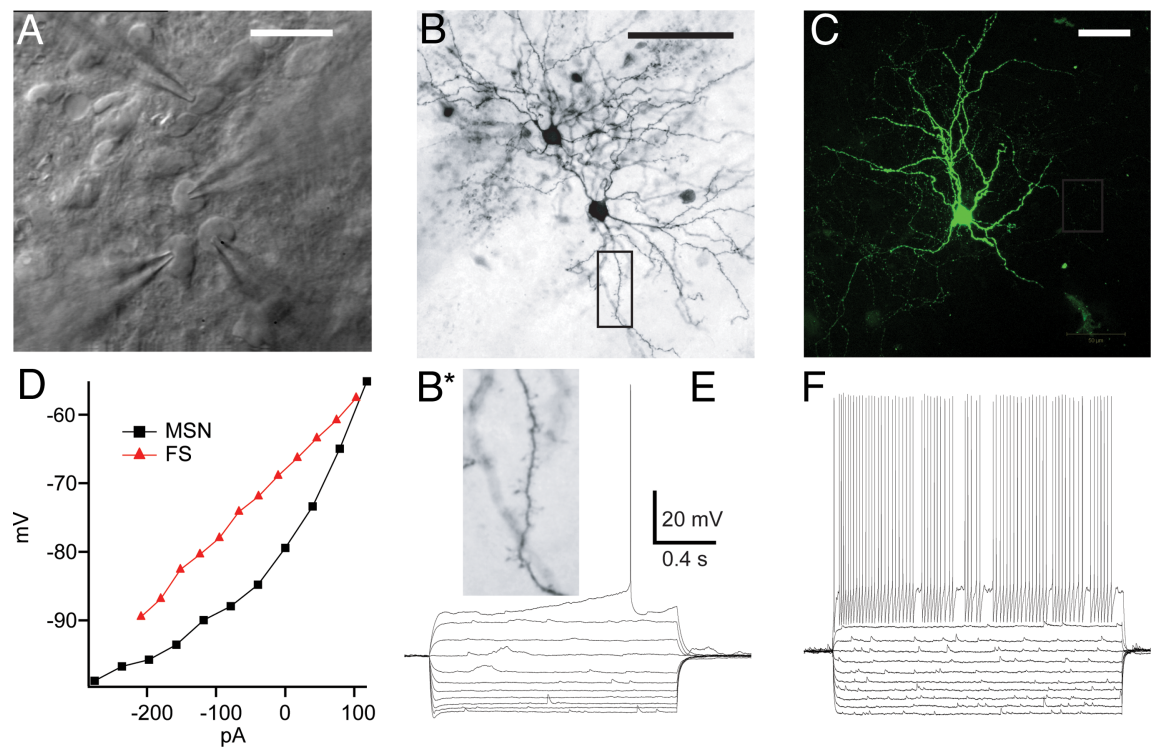

Figure 1. Patch recordings from MSN and FS striatal neurons. $A$, Infrared microscopy image of a multineuron patch experiment. $\boldsymbol{B}$, Biocytin-filled pair of MSNs (rat; $18 \mathrm{~d}$ of age) stained for light microscopy. The enlarged image $\left(\boldsymbol{B}^{*}\right)$ shows dendritic spines characteristic of MSNs. C, FS interneuron, biocytin loaded and stained for fluorescent microscopy with aspiny beaded dendrites (rat; $15 \mathrm{~d}$ of age). D, Example of the current-voltage relationship obtained from an MSN (black squares) and FS cell (red triangles). Note the rectification in the MSN, apparent from the change in curve slope. $\boldsymbol{E}$, Response of an MSN to increasing step current injections showing the rectification in hyperpolarized steps, as well as characteristic ramp and delay preceding the action potential. $\boldsymbol{F}, A$ typical response of an $\mathrm{FS}$ cell to the same stimulation protocol as in $\boldsymbol{E}$, showing high discharge rate, nonaccommodating discharge pattern, and the fast and deep afterhyperpolarization. Scale bars, $25 \mu \mathrm{m}$.

Jackson ImmunoResearch Laboratories) diluted in $0.1 \mathrm{M}$ PB containing $1 \%$ Triton $\mathrm{X}-100$. In between all experimental procedures, slices were washed with $0.01 \mathrm{M}$ PBS. Finally, they were placed on slides, let to dry, and thereafter mounted with glycerol containing 2.5\% diazabicyclooctane (Sigma-Aldrich) and stored at $-20^{\circ} \mathrm{C}$.

\section{Results}

\section{Classification of recorded neurons}

We recorded simultaneously from pairs, triplets, and quadruplets of neighboring (within $100 \mu \mathrm{m}$ lateral somatic distance) striatal neurons (Fig. $1 A-C$ ). The first set of experiments was performed in rat striatal slices, in which neurons were not fluorescently labeled; therefore, differentiation could be done only between MSNs and interneurons. To differentiate between direct- and indirect-pathway MSNs, we then used two complementary methods, fluorescent retrograde labeling in rats and slices from BAC transgenic EGFP mice. Classification of MSNs or FS cells was done according to their electrical properties and, when performed, morphological staining. MSNs and FS cells were unambiguously distinguished from each other by their voltage responses to depolarizing and hyperpolarizing current steps (Kawaguchi et al., 1995; Koós and Tepper, 1999; Taverna et al., 2007). The majority of recorded neurons were MSNs, characterized by a hyperpolarized resting membrane potential, strong inward rectification (Fig. $1 D, E$ ), depolarizing ramp response for near-threshold current steps, typical discharge response (Fig. $1 E$ ), and spiny dendrites (Fig. $1 B$ ). FS cells were characterized by their nonaccommodating or "stuttering" (Gupta et al., 2000) discharge patterns (Fig. $1 F$ ), high discharge rate, fast and deep afterhyperpolarization, narrow APs, and aspiny dendrites (Fig. 1C).

\section{Synaptic connections onto MSNs in rat striatum}

Synaptic connectivity was examined for all recorded neuron pairs by evoking APs in presynaptic neurons by brief current injections (see Materials and Methods) and recording the responses in the other simultaneously recorded neurons. Synaptic responses in postsynaptic MSNs were measured at a membrane potential near $-80 \mathrm{mV}$, both in current and voltage clamp. Synaptic responses were recorded within the first $30 \mathrm{~min}$ after achieving a whole-cell recording, and in most cases no apparent rundown was observed within that time frame. In rat slices, connectivity between MSNs was sparse (20\%; $n=40$ of 202 of tested MSN pairs) compared with connections from FS cells to MSNs ( $74 \% ; n=29$ of 39). Apart from a single case of very weak electrical coupling (coupling coefficient, $<0.3 \%$ ), all connections formed onto MSNs were unidirectional. Synaptic strength differed between the two types of connections, measured as the amplitude of the first synaptic response (PSP) in the train (Fig. 2). FS $\rightarrow$ MSN connections were more than three times larger than MSN $\rightarrow$ MSN ones, with an average amplitude of $1.52 \mathrm{mV}( \pm 2.37$; ranging from 0.1 to $7.7 \mathrm{mV} ; n=31)$ compared with $0.45 \mathrm{mV}( \pm 0.27$; range, $0.07-1.21$ $\mathrm{mV} ; n=23 ; p=0.02$, Student's $t$ test) (Fig. 2C, Table 1). To assess differences in release probability between the two pathways, we performed a paired-pulse analysis, in which the amplitude ratio of the second and first responses in the train was calculated for a $50 \mathrm{~ms}$ interval. Both pathways displayed on average paired-pulse depression; however, in MSN $\rightarrow$ MSN connections, the range of paired-pulse ratios was much larger than in $\mathrm{FS} \rightarrow \mathrm{MSN}$ connections ( $0.88 \pm 0.54$; range, $0.28-2.6$; compared with $0.64 \pm 0.20$, ranging $0.25-0.90$, respectively) (Fig. $2 D$, Table 1 ). Paired-pulse facilitation was observed in seven MSN $\rightarrow$ MSN connections (see example in Fig. $2 \mathrm{~A}$, middle trace) but never in $\mathrm{FS} \rightarrow \mathrm{MSN}$ connections.

\section{Differential synaptic dynamics}

The variability observed in the paired-pulse properties of $\mathrm{MSN} \rightarrow$ MSN connections suggested that, in these synapses, facilitation and depression take place simultaneously and underlie the observed dynamics. To extract these properties, we used a phenomenological model for synaptic dynamics that captures a wide range of synaptic activity dependence (Markram et al., 1998; Tsodyks et al., 1998) and is relatively simple to apply to experimental data (see Materials and Methods). The commonly used protocol for this analysis is an AP train followed by a recovery test pulse (see Materials and Methods) (Fig. 3). Interestingly, the recovery test pulse revealed a large difference between the two pathways, in which connections between MSNs displayed strong facilitation ( $>50 \%$ increase) compared with the depression observed in FS $\rightarrow$ MSN synapses (Fig. 3C,D). The facilitation ratio between the recovery response and the first PSP in MSN $\rightarrow$ MSN connections was 1.51 ( \pm 1.1 ; ranging between 0.58 and 5.37 ). Recovery response facilitation was observed in most (22 of 31) of these connections, in sharp contrast to the FS $\rightarrow$ MSN connections, which displayed depression in all cases (amplitude ratio, $0.63 \pm$ 0.2 ; range, $0.24-0.95 ; n=23$ ). The facilitation of the recovery test response was present at different train frequencies (Fig. $3 A$ ) 
and at both current- and voltage-clamp recording configurations (Fig. $3 B$ ). We then used the measured synaptic amplitudes to extract the model parameters quantifying the synaptic dynamics (see Materials and Methods), in particular the time constants for facilitation $(F)$ and depression $(D)$. The relationship between these two time constants determines whether a synapse is depressing $(D>F)$, facilitating $(F>D)$, or is governed by both processes $(D \approx F)$. The respective time constants were extracted for all analyzed synapses and showed clear separation between the two types of synapses (Fig. 3E). Whereas FS $\rightarrow$ MSN synapses were purely depressing, with a very short $F$ ( $53 \pm 53 \mathrm{~ms}$, ranging from 1 to $160 \mathrm{~ms}$ ), a much longer $D(902 \pm 954 \mathrm{~ms}$; range, $238-4332 \mathrm{~ms}$ ), and a small $F / D$ ratio of $0.16, \mathrm{MSN} \rightarrow \mathrm{MSN}$ synapses had a clear facilitatory component alongside depression, as evident from their respective time constants $(F, 859 \pm 1009 \mathrm{~ms} ; D, 222 \pm$ $189 \mathrm{~ms}$ ) and the high $F / D$ ratio of 4.76 (Fig. $3 F$ ). The dynamics of the different connections was similar when recorded in voltage- or current-clamp modes, with apparent depression in FS-MSN connections, and a facilitatory component in MSN-MSN connections (Fig. 3B). MSN-MSN connections differed only slightly between the two recording configurations, with a decrease in the recovery test response (RTR) facilitation ratio in current traces $(1.08 \pm 0.28 ; 0.99 \pm$ $0.32 ; n=6)$. Our data show that MSN-MSN connections are sparser, smaller, and more diverse than connections from FS to MSNs. One possible explanation for this diversity is the division of MSNs into two differently projecting subpopulations, namely, the striatonigral and striatopallidal MSNs.

To identify MSNs according to their projection type, we used two alternative methods, retrograde labeling of striatonigral MSNs with fluorescent beads and transgenic EGFP mice (see Materials and Methods) (Fig. 4). In retrogradely labeled rat slices, we simultaneously recorded from labeled (dMSN, for putative direct-pathway MSNs) and nearby nonlabeled (iMSN, for putative indirect-pathway MSNs) neurons (Fig. 4A-C). Retrogradely labeled neurons were all MSNs, as verified by their electrical properties. In retrogradely labeled slices, we recorded from a total of 83 pairs, 8 of which had synaptic connections ( 6 connections between MSNs and 2 from FS to MSNs). Both MSN types also received synaptic connections from presynaptic iMSNs (iMSN $\rightarrow$ iMSN, 2 of 14 ; iMSN $\rightarrow$ dMSN, 3 of 10 ), and 1 connection was found between dMSNs (dMSN $\rightarrow$ dMSN, 1 of 13). From the six MSN-MSN connections, four had facilitation of the test response, and two were depressing connections (average amplitude ratio, $110 \pm 45 \% ; n=6$ ). Labeled and nonlabeled MSNs received synapses from FS cells (one of one FS $\rightarrow$ dMSN and one of one FS $\rightarrow$ iMSN connected pairs), both of which were depressing connections, with a clear decrease in the amplitude of the recovery test response ( 67 and $77 \%$ of first PSP, respectively).

\section{Connectivity of identified MSNs in mouse striatum}

As an additional independent method for identifying MSN subpopulations, we used transgenic mice expressing EGFP in dMSNs
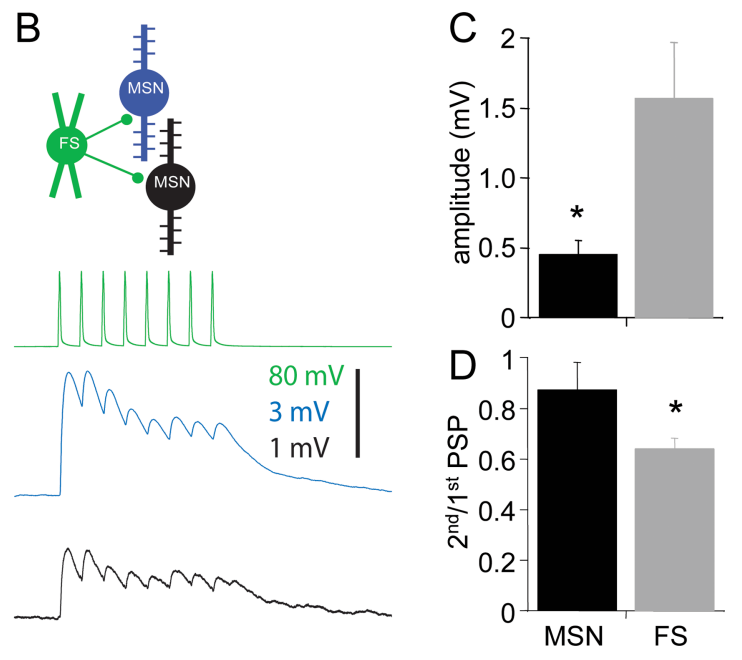

Figure 2. Synaptic connections formed by MSNs and FS cells onto MSNs. $\boldsymbol{A}$, Two examples of synaptic connectivity between MSNs. The presynaptic MSN was stimulated with a $20 \mathrm{~Hz}$ train of action potentials (top trace; black) and postsynaptic responses (bottom traces; blue) were recorded in postsynaptic MSNs. The middle trace shows a facilitating synaptic response, whereas the $n=23$ ). The difference was significant, with $p=0.02$, Student's $t$ test. $\boldsymbol{D}$, Paired-pulse depression in the respective pathways, as calculated from the amplitudes of the first and second responses ( $p=0.03, t$ test). The average paired-pulse ratio of MSN-MSN pairs was larger because of the occurrence of depressing and facilitating synapses, which were absent in FS-MSN connections. bars indicate SEM.

(Wang et al., 2006b). We recorded from labeled and nonlabeled neighboring neurons in slices of $\mathrm{D}_{1}$-EGFP mice (see Materials and Methods) (Fig. 4D-F) and found synaptic interactions in 23 of 294 MSN-MSN tested connections (Fig. 5). From these MSN-MSN connections, a majority (74\%; 17 of 23 ) was from presynaptic iMSNs and 6 connections (26\%) had a presynaptic dMSN (for synaptic properties, see Table 1). The connections from dMSNs did not have significantly different amplitudes than those from iMSNs $(0.28$ and $0.39 \mathrm{mV}$, respectively; $p=0.55, t$ test). These connections displayed recovery test facilitation $(110 \pm 47 \%$; ranging from 62 to $170 \% ; n=6$ ), with two (of three) facilitating dMSN $\rightarrow$ iMSN connections and one facilitating $\mathrm{dMSN} \rightarrow \mathrm{dMSN}$ connection (the three others were depressing synapses).

iMSNs formed connections onto dMSNs $(13 \% ; n=10$ of 80 pairs) and iMSNs (23\%; $n=7$ of 31 pairs), both of which had depressing and facilitating synapses (Fig. $5 A$ ). The connections from iMSNs onto the two types of target cells were not significantly different in their amplitude $(0.27 \pm 0.09$ and $0.45 \pm 0.44$ $\mathrm{mV}$, respectively; $p=0.17, t$ test), paired-pulse ratio (of $111 \pm 59$ and $95 \pm 48 \% ; p=0.67$ ), recovery test facilitation (151 \pm 64 and $139 \pm 69 \% ; p=0.98$ ) (Fig. $5 D$ ), as well as the utilization factor ( $U)(0.36 \pm 0.18$ and $0.34 \pm 0.19 ; p=0.82)$. In these connections, with presynaptic iMSNs, the type of postsynaptic MSNs did not determine the dynamics of the synaptic connection, as we saw in two cases in which a dMSN received both a depressing and a facilitating connection from two different iMSNs (Fig. 5A). In two separate cases, the same presynaptic iMSN contacted a dMSN and an iMSN with facilitating synapses, showing that the individual presynaptic MSN, but not the MSN type, determined the synaptic dynamics of the connection (see example in Fig. 5A).

\section{Homogenous inhibition of both MSN subpopulations by FS cells}

As in rat striatum, $\mathrm{FS} \rightarrow \mathrm{MSN}$ connectivity recorded in mice had higher prevalence than MSN $\rightarrow$ MSN connectivity $(78 \%$, 
Table 1. Properties of synaptic connections between MSNs and FS cells

\begin{tabular}{|c|c|c|c|c|c|c|c|}
\hline & $\begin{array}{l}\text { Connection } \\
\text { probability }(\% ; n / N \text { pairs) }\end{array}$ & Amplitude (mV) & $\begin{array}{l}\text { Paired-pulse ratio } \\
\text { (2nd/1st response) }\end{array}$ & $\begin{array}{l}\text { Recovery test ratio } \\
\text { (RTR/1st response) }\end{array}$ & $\begin{array}{l}\text { Depression time } \\
\text { constant (D) (ms) }\end{array}$ & $\begin{array}{l}\text { Facilitation time } \\
\text { constant }(F)(\mathrm{ms})\end{array}$ & $\begin{array}{l}\text { Synaptic utilization } \\
\text { factor }(U)\end{array}$ \\
\hline \multicolumn{8}{|l|}{ Rat } \\
\hline MSN-MSN & $20 \% ; 40 / 202$ & $0.45 \pm 0.27$ & $0.88 \pm 0.54$ & $1.51 \pm 1.10$ & $222 \pm 189$ & $859 \pm 1009$ & $0.42 \pm 0.21$ \\
\hline FS-MSN & $74 \% ; 29 / 39$ & $1.52 \pm 2.37$ & $0.64 \pm 0.20$ & $0.63 \pm 0.2$ & $902 \pm 954$ & $53 \pm 53$ & $0.29 \pm 0.19$ \\
\hline \multicolumn{8}{|l|}{ GFP mice } \\
\hline D1-D1 & $7 \% ; 3 / 43$ & $0.24 \pm 0.15$ & $0.91 \pm 0.63$ & $1.23 \pm 0.50$ & $192 \pm 114$ & $1266 \pm 1427$ & $0.39 \pm 0.22$ \\
\hline D1-D2 & $4.5 \% ; 3 / 66$ & $0.33 \pm 0.15$ & $0.84 \pm 0.30$ & $1.16 \pm 0.29$ & $96 \pm 9$ & $313 \pm 363$ & $0.46 \pm 0.24$ \\
\hline D2-D1 & $13 \% ; 10 / 80$ & $0.27 \pm 0.09$ & $1.1 \pm 0.6$ & $1.51 \pm 0.64$ & $365 \pm 471$ & $570 \pm 783$ & $0.36 \pm 0.18$ \\
\hline D2-D2 & $23 \% ; 7 / 31$ & $0.45 \pm 0.44$ & $0.95 \pm 0.48$ & $1.39 \pm 0.69$ & $149 \pm 90$ & $1462 \pm 1800$ & $0.34 \pm 0.19$ \\
\hline FS-D1 & $89 \% ; 8 / 9$ & $4.8 \pm 4.9$ & $0.62 \pm 0.12$ & $0.72 \pm 0.08$ & $740 \pm 350$ & $3.1 \pm 2.4$ & $0.24 \pm 0.07$ \\
\hline FS-D2 & $67 \% ; 6 / 9$ & $3.1 \pm 4.1$ & $0.66 \pm 0.14$ & $0.63 \pm 0.19$ & $850 \pm 500$ & $4.5 \pm 2.7$ & $0.23 \pm 0.07$ \\
\hline
\end{tabular}

The table presents a summary of the properties of synaptic connections formed between MSNs and FS cells. Data are presented as mean \pm SD.

A
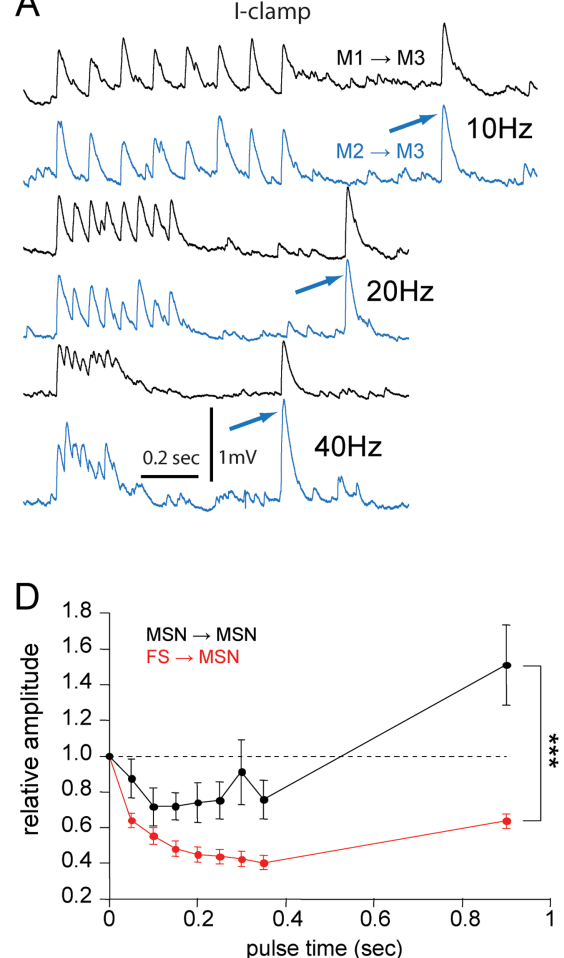

B
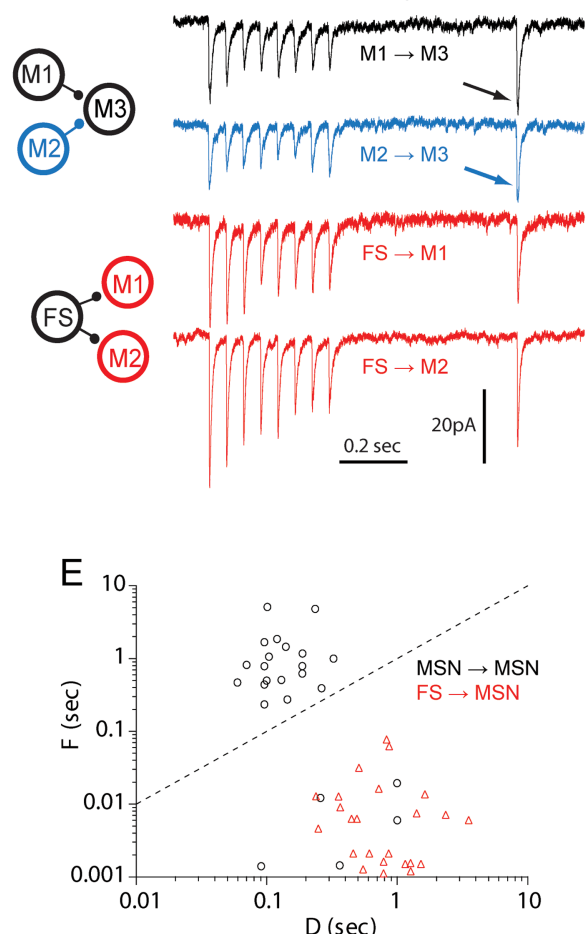

C

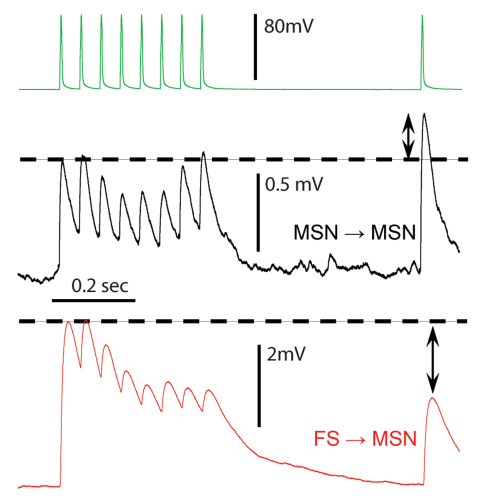

$\mathrm{F}$

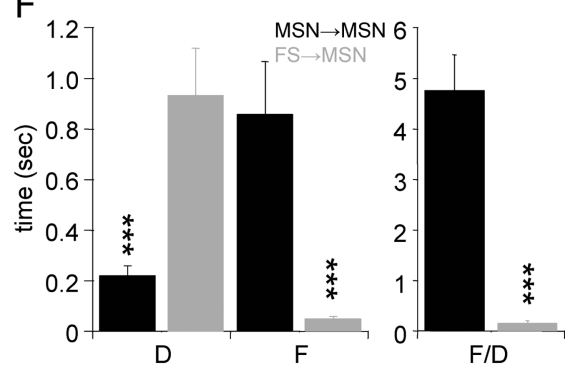

Figure 3. Differential synaptic dynamics of feedback and feedforward connections. A, An example of converging MSN-MSN connectivity. A postsynaptic MSN (M3) received direct connections from two neighboring MSNs (M1 and M2). The connection was tested in different train frequencies (10, 20, $40 \mathrm{~Hz}$ trains of 8 action potentials), showing frequency-dependent depression and facilitation. Note the increase in amplitude of the recovery test response (denoted by blue arrows), revealing the underlying facilitatory component. $\boldsymbol{B}$, Examples of divergent synaptic connectivity from FS to two neighboring MSNs as recorded in voltage clamp (red traces, bottom). Note the depression of the recovery test response after $0.55 \mathrm{~s}$ after a $20 \mathrm{~Hz}$ train. The top traces are of the same connections as in $\boldsymbol{A}$, acquired in voltage-clamp mode. C, MSNs receive different types of input from neighboring MSNs and FS cells. Two examples of synaptic responses to $20 \mathrm{~Hz}$ trains (green) and a recovery response are depicted. Note the facilitation of the recovery response in the MSN $\rightarrow$ MSN connection (black) compared with the depression of the FS $\rightarrow$ MSN response (red). $D$, Average responses of all analyzed connections normalized to the amplitude of the first PSP (FS-MSN connections in red, $n=23$; MSN-MSN in black, $n=31$ ). $E$, Facilitation and depression time constants of all analyzed connections are plotted against each other in a logarithmic plot. The dashed line represents the $F=D$ curve, showing that all FS $\rightarrow$ MSN, but not all MSN $\rightarrow$ MSN connections, were depressing. $F$, The synaptic dynamics of the two connection types are significantly different, as seen by the values of the time constants for facilitation $(F)$ and depression $(D)$, and the ratio (right bar graph). ${ }^{* *} p<0.001$, Student's $t$ test. Error bars indicate SEM.

14 of 18; compared with $10 \%, 23$ of 227), larger amplitude $(4.21 \pm 3.9 \mathrm{mV} ; 0.41 \pm 0.35 \mathrm{mV} ; p<0.001$, Student's $t$ test $)$, and displayed only depressing dynamics. The high connection probability, combined with the homogeneity in dynamic properties, suggested that both direct- and indirect-pathway MSNs are similarly inhibited by FS interneurons. Indeed, both iMSNs $(67 \% ; n=6$ of 9$)$ and dMSNs $(89 \% ; n=8$ of 9$)$ received inhibitory connections from FS cells with very similar dynamic properties. Moreover, in two experiments, we found individual FS cells forming divergent connections on both MSN types (one example depicted in Fig. 6A), further suggest- ing unspecific inhibition mediated by FS cells. The synaptic properties of $\mathrm{FS} \rightarrow \mathrm{dMSN}$ and $\mathrm{FS} \rightarrow \mathrm{iMSN}$ were not significantly different in terms of their amplitudes $(4.8 \pm 4.9, n=8$; and $3.1 \pm 4.1, n=6)$, paired-pulse depression $(62 \pm 12$ and $66 \pm 14 \%)$, recovery test response (72 \pm 8 and $63 \pm 19 \%)$, and dynamic model parameters $(U, 0.24 \pm 0.07$ and $0.23 \pm 0.07$; $D, 0.74 \pm 0.35$ and $0.85 \pm 0.5 \mathrm{~s} ; F, 3.1 \pm 2.4$ and $4.5 \pm 2.7 \mathrm{~ms})$ (Fig. 6B,C, Table 1). These data show that both MSN subpopulations receive ubiquitous and homogeneous inhibition from FS cells, which is, moreover, mediated by the same pool of presynaptic interneurons. 

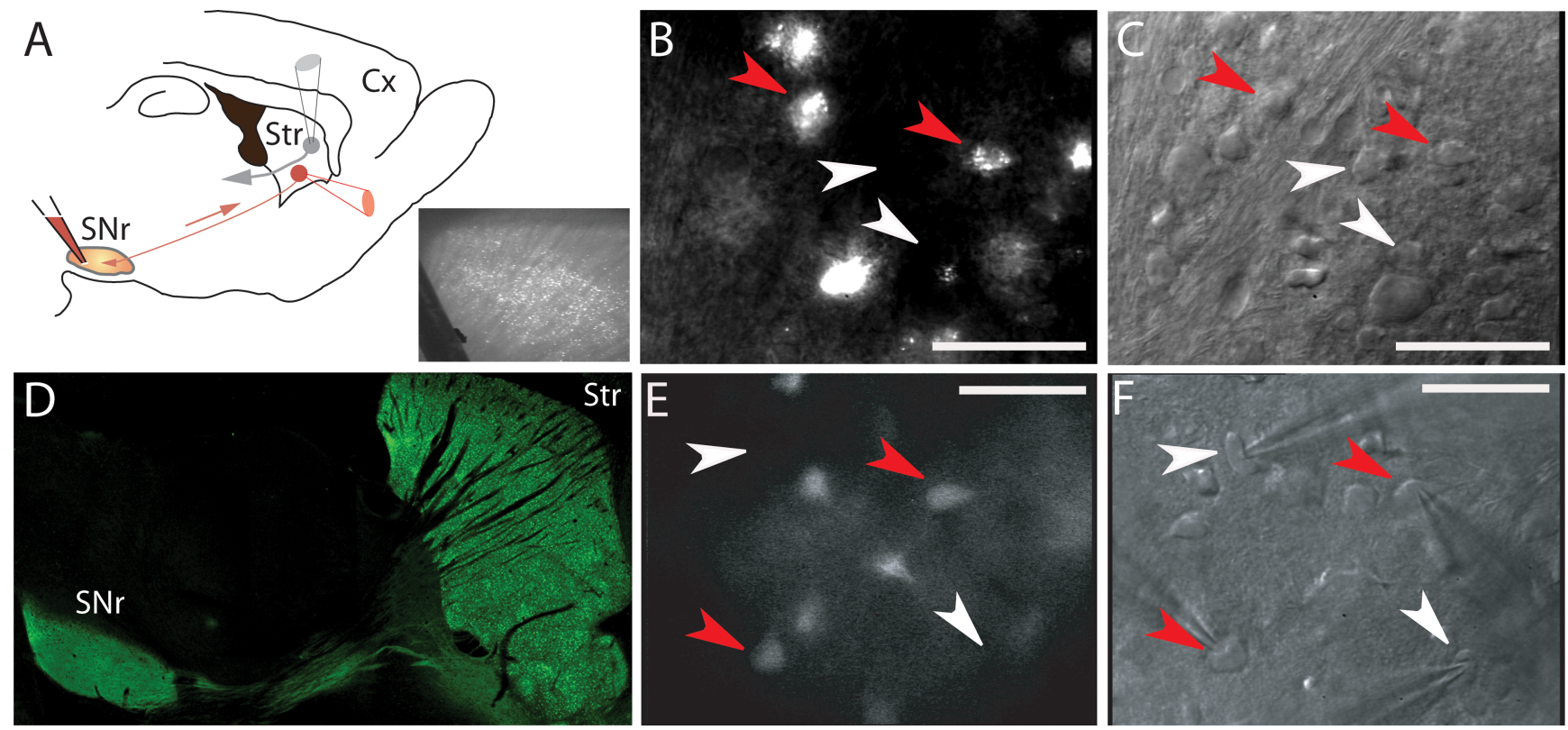

Figure 4. Fluorescent identification of direct-pathway MSNs. A, Retrograde labeling of rat striatonigral MSNs by injection of fluorescent beads into the substantia nigra pars reticulata. The beads are transported by the axons to the cell bodies and can then be visualized under fluorescence microscopy. $\boldsymbol{B}$, Individual striatal neurons after retrograde labeling. The red arrows designate retrogradely labeled striatonigral MSNs, and the white arrows show the position of unlabeled neurons, which are MSNs or interneurons. $\boldsymbol{C}$, The same neurons as in $\boldsymbol{B}$ under

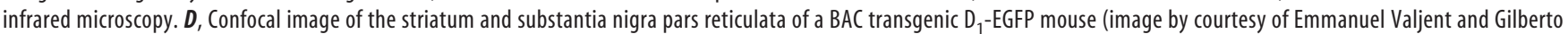
Fisone, Karolinska Institute, Stockholm, Sweden). $\boldsymbol{E}$, Individual striatal neurons in slices of EGFP mouse. The labeled neurons are visible under epifluorescence microscopy and can be selected for recording. $\boldsymbol{F}$, The same neurons as in $\boldsymbol{E}$, under IR microscopy. The red arrows designate the fluorescent neurons selected to be recorded under IR optics. Scale bars, $50 \mu \mathrm{m}$. $\mathrm{SNr}$, Substantia nigra pars reticulata; Str, striatum; Cx, cortex.

\section{Discussion}

In this study, we showed fundamental differences in the dynamic properties of two inhibitory pathways within the striatal microcircuitry. Inhibition by FS cells is robust, homogenous, and exerted by the same FS cells onto both striatonigral and striatopallidal projection neurons. In contrast, MSN-MSN connectivity is sparser, weaker, and exhibits diverse activity-dependent synaptic dynamics. The connectivity prevalence between MSNs depended on the type of presynaptic MSN; however, the dynamic properties were not determined by the type of interconnected MSNs. The differences between the FS $\rightarrow$ MSN and MSN $\rightarrow$ MSN connectivity are clear in both species and throughout different developmental stages, suggesting that they indeed constitute a basic organizational principle in the striatal microcircuitry.

\section{Robust feedforward inhibition from FS cells onto MSNs}

Inhibition mediated by FS cells onto MSNs had very different dynamics than that observed between MSNs. FS $\rightarrow$ MSN synaptic connections mediated large, depressing responses with negligible facilitation, which were strikingly homogenous across different target cells. We show that both neighboring directand indirect-pathway MSNs are inhibited by FS cells, with similar amplitudes and dynamics. It is still not clear whether and to what degree FS cells have preferences for any of the MSN projection types; however, as exemplified in our data, the same FS cells target MSNs of both projection types (Fig. $6 A)$. FS cells therefore may not selectively inhibit one striatal projection or the other, but rather efficiently and similarly inhibit a large fraction of their neighboring MSNs of both types. The depressing nature of the FS $\rightarrow$ MSN synapse makes it tuned to faithfully transmit the onset of FS activity, enabling FS cells to mediate potent synchronized inhibition after cortical excitation (Mallet et al., 2005; but see Berke, 2008). The role of such inhibition by FS cells may be in preventing target MSNs from discharge after excitatory input, but it may also be effective in synchronizing the output of target MSNs without necessarily silencing them completely, but rather by delaying discharge (Koós and Tepper, 1999). Our results suggest that both types of inhibition are very different in terms of their respective dynamics regardless of the postsynaptic MSN type. This was also shown to be the case for glutamatergic input to MSNs, in which the dynamics of thalamic input was very different from that of cortical input, for both striatopallidal and striatonigral MSNs (Ding et al., 2008).

$\mathrm{FS} \rightarrow$ MSN connectivity was severalfold more prevalent than MSN-MSN connectivity, in accord with previous reports (Koós and Tepper, 1999; Koós et al., 2004; Gustafson et al., 2006; Taverna et al., 2007; Tecuapetla et al., 2007). This big difference in connectivity can be partly attributed to the electrotonic attenuation of synaptic responses at distal dendrites compared with the little attenuation of perisomatic inputs from FS cells (Tepper et al., 2008). However, even in experiments in which high intracellular chloride concentrations were used to improve the detection of GABAergic inputs (Koós et al., 2004; Taverna et al., 2008; Tecuapetla et al., 2009), feedback connectivity was sparse. Interestingly, a similar connectivity pattern is found in the neocortical microcircuit, in which projection neurons (pyramidal cells) also constitute the vast majority of neurons and are sparsely interconnected (Markram et al., 1997), whereas connectivity from GABAergic FS interneurons onto pyramidal cells is severalfold higher (Holmgren et al., 2003). This suggests that FS cells in the respective microcircuits, in addition to their similar intrinsic properties (Kawaguchi, 1995; Kawaguchi et al., 1995), share similar network properties and function in mediating feedforward inhibition to their respective principal targets. 

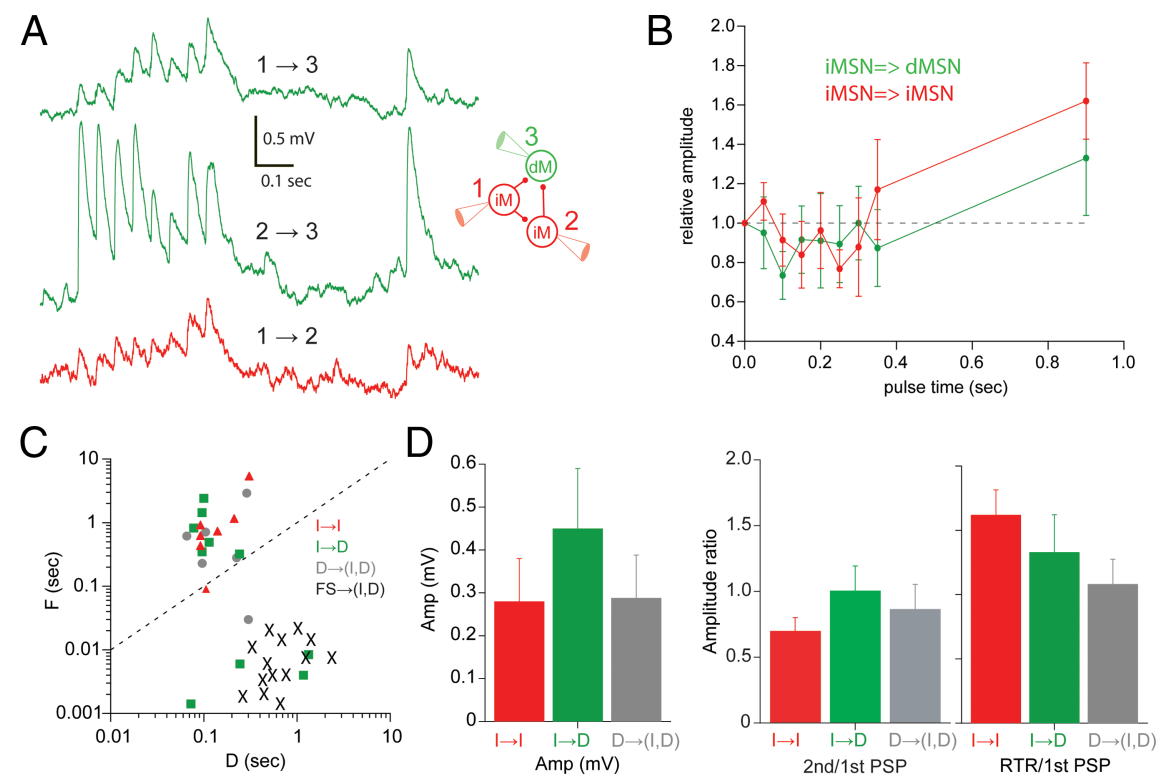

Figure 5. Synaptic connections between EGFP-identified MSNs. A, A network of neighboring MSNs in ventral striatum displaying divergent and convergent synaptic connections. Cell 3 is a direct-pathway MSN (dM) receiving convergent input from two indirect MSNs (iM) with different dynamic properties. Note the facilitation in the $1 \rightarrow 3$ connection (top trace) compared with the depressing connection received by cell 3 , and the facilitating connections onto both targets of cell 1 . $\boldsymbol{B}$, Average amplitudes, normalized to the amplitude of the first PSP, for connections from iMSNs onto dMSNs (in green; $n=10$ ) and iMSNs (in red; $n=7$ ). Note the facilitation of the recovery test response, which is absent in FS $\rightarrow$ MSN connections (Figs. 3, 6). C, Facilitation and depression time constants of connections between identified MSNs are plotted against each other in a logarithmic plot. $\mathrm{iMSN} \rightarrow$ iMSN connections are marked with red triangles, iMSN $\rightarrow$ dMSN in green squares, presynaptic dMSN connections in gray circles, and FS $\rightarrow$ MSN connections in black "X." D , Synaptic properties of connections from presynaptic iMSNs did not significantly differ according to the postsynaptic MSN type and were not significantly different from connections between MSNs in which presynaptic neurons were dMSNs. Error bars indicate SEM.
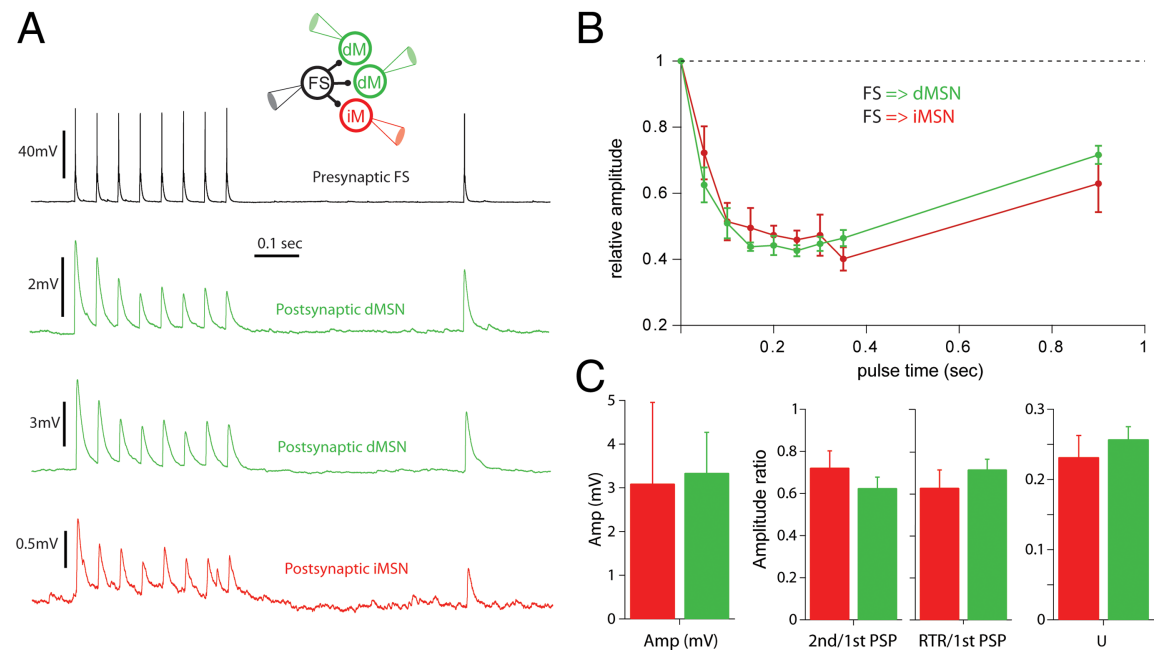

Figure 6. Synaptic connections from FS cells onto both types of MSNs. A, An example of a divergent connection from a single FS cell onto three target MSNs of different projection types. Note the similarity in the dynamics of the responses on the different MSN types. $\boldsymbol{B}$, Average amplitudes normalized to the amplitude of the first PSP, for connections from FS onto iMSNs (in red; $n=6$ ) and dMSNs (in green; $n=9$ ). C, Synaptic properties of connected FS cells did not differ significantly according to the postsynaptic MSN type. Both $\boldsymbol{B}$ and $C$ show the high degree in homogeneity in the FS $\rightarrow$ MSN pathway onto both projection types of MSNs. Error bars indicate SEM.

\section{Connectivity between MSNs of different types}

Synaptic connections between MSNs of both types displayed a large diversity in terms of the dynamic properties, with cases of depression, facilitation, and mixed responses in which synaptic depression was followed by facilitation of the recovery test response (Fig. 3A). This was in sharp contrast to the homogenous dynamics of FS $\rightarrow$ MSN connections. This variability in dynamics was, surprisingly, not accounted for by the projection identity of the presynaptic and postsynaptic neurons, since all four possible combinations (presynaptic and postsynaptic iMSN/dMSN) had cases of facilitating as well as depressing synapses (Fig. 5). One aspect of MSN-MSN connectivity that did, however, depend on the presynaptic MSN subtype was the prevalence of connectivity. As reported recently (Taverna et al., 2008), we also observed a nonrandom organization of MSN interconnectivity, in which synapses formed by iMSNs seemed to be more common than those formed by presynaptic dMSNs. The reasons for this difference are still not clear but may be related to properties of the axonal arborization and the dendritic targeting preferences of the respective types. The fact that these differences were observed both in rats as well as $\mathrm{D}_{1}$-EGFP mice suggests that it is not an artifact attributable to the particular transgenic mouse used. Whereas the projection type of connected MSNs did not determine the synaptic dynamics, individual presynaptic neurons induced similar response dynamics in their targets, as seen in divergent connections, both from presynaptic MSNs and FS cells (Figs. 5, 6). Interestingly, this is not the typical scenario in other microcircuits, in which the same presynaptic neuron may induce drastically different synaptic responses in different types of postsynaptic targets (Thomson et al., 1993; Markram et al., 1998; Reyes et al., 1998).

\section{Variability in the dynamics of MSN-MSN synaptic transmission} Unlike the very dominant facilitation observed at certain types of cortical synapses (Thomson et al., 1993), the facilitation in $\mathrm{MSN} \rightarrow \mathrm{MSN}$ connections was commonly masked by a simultaneously occurring depressing component. The interplay between these processes is likely to cause the variability we observed in $\mathrm{MSN} \rightarrow \mathrm{MSN}$ connectivity and may also underlie previous reports of both facilitation and depression mediated by presynaptic MSNs (Czubayko and Plenz, 2002; Venance et al., 2004; Rav-Acha et al., 2005; Gustafson et al., 2006). One possible explanation for the observed variability is the postsynaptic dendritic location of MSN-MSN synapses (Wilson and Groves, 1980; Somogyi et al., 1981; Tepper et al., 2008). Unlike FS-MSN synapses, which target a closer and more confined perisomatic region, synapses between MSNs target dendrites with variable thickness and at various electrotonic distances from the soma and dendritic branching points. 
The dendritic locus of MSN-MSN inhibition enables it to affect the nonlinear processes of dendritic integration as well as the plasticity of nearby excitatory inputs, which also target MSN dendrites (Plenz, 2003; Carter et al., 2007; Wilson, 2007; Tepper et al., 2008). In addition to these functional properties, the dendritic location also shapes the apparent dynamics of the synaptic responses (Banitt et al., 2005). Synaptic activation causes a local conductance increase as well as reduction of the synaptic driving force, both of which induce a depressing component in synaptic responses. This "apparent depression" strongly depends on the location of the synaptic contact and is likely to contribute to the variability we observed in synaptic dynamics.

Similar recovery test facilitation was observed in other neural microcircuits in the prefrontal cortex (Wang et al., 2006a) and in the hippocampus (Hefft and Jonas, 2005). As in the MSN-MSN connections we observed, responses typically displayed depression during the burst of action potentials, but facilitated after a pause of several hundreds of milliseconds. This facilitation was observed both in current- and voltage-clamp modes, and was also observed when postsynaptic neurons were recorded in a cesiumbased pipette solution, suggesting that it is mainly dictated by presynaptic processes. The biophysical mechanisms underlying the observed synaptic dynamics were not explored in this study; however, it is predicted that protocols affecting the release process such as changing extracellular calcium concentrations or loading the presynaptic neurons with $\mathrm{CsCl}$ (Hull et al., 2009) would significantly alter the dynamics of these connections. Presynaptic calcium channels are subject to multiple modulatory processes that may underlie the various forms of synaptic facilitation (for review, see Catterall and Few, 2008).

The variability in synaptic dynamics in MSN-MSN connections was not explained by the type of connected neurons but it may reflect intrinsic variability in both presynaptic and postsynaptic elements. In our study, we did not record neuronal positions with respect to the patch and matrix striatal compartments, which may also contribute to the observed variability in dynamics, as recently suggested for synaptic strength (Tecuapetla et al., 2009).

\section{Summary}

Our study shows that MSN $\rightarrow$ MSN and FS $\rightarrow$ MSN inhibitory pathways in the striatum have fundamentally different functional properties, with highly homogenously depressing, reliable inhibition from FS onto both projection pathways, and sparse and variable interconnectivity among MSNs of both direct and indirect projection systems. These results suggest that the functional role of inhibition from FS cells might be not in separating the activity of the two striatal projections, but rather in shaping striatal output for both.

\section{References}

Banitt Y, Martin KA, Segev I (2005) Depressed responses of facilitatory synapses. J Neurophysiol 94:865-870.

Barnes TD, Kubota Y, Hu D, Jin DZ, Graybiel AM (2005) Activity of striatal neurons reflects dynamic encoding and recoding of procedural memories. Nature 437:1158-1161.

Bennett BD, Bolam JP (1994) Synaptic input and output of parvalbuminimmunoreactive neurons in the neostriatum of the rat. Neuroscience 62:707-719.

Berke JD (2008) Uncoordinated firing rate changes of striatal fast-spiking interneurons during behavioral task performance. J Neurosci 28:1007510080 .

Bevan MD, Booth PA, Eaton SA, Bolam JP (1998) Selective innervation of neostriatal interneurons by a subclass of neuron in the globus pallidus of the rat. J Neurosci 18:9438-9452.

Carter AG, Soler-Llavina GJ, Sabatini BL (2007) Timing and location of synaptic inputs determine modes of subthreshold integration in striatal medium spiny neurons. J Neurosci 27:8967-8977.

Catterall WA, Few AP (2008) Calcium channel regulation and presynaptic plasticity. Neuron 59:882-901.

Czubayko U, Plenz D (2002) Fast synaptic transmission between striatal spiny projection neurons. Proc Natl Acad Sci U S A 99:15764-15769.

Day M, Wokosin D, Plotkin JL, Tian X, Surmeier DJ (2008) Differential excitability and modulation of striatal medium spiny neuron dendrites. J Neurosci 28:11603-11614.

Ding J, Peterson JD, Surmeier DJ (2008) Corticostriatal and thalamostriatal synapses have distinctive properties. J Neurosci 28:6483-6492.

Graveland GA, Difiglia M (1985) The frequency and distribution of medium-sized neurons with indented nuclei in the primate and rodent neostriatum. Brain Res 327:307-311.

Graybiel AM, Aosaki T, Flaherty AW, Kimura M (1994) The basal ganglia and adaptive motor control. Science 265:1826-1831.

Grillner S, Hellgren J, Ménard A, Saitoh K, Wikström MA (2005) Mechanisms for selection of basic motor programs-roles for the striatum and pallidum. Trends Neurosci 28:364-370.

Gupta A, Wang Y, Markram H (2000) Organizing principles for a diversity of GABAergic interneurons and synapses in the neocortex. Science 287:273-278.

Gustafson N, Gireesh-Dharmaraj E, Czubayko U, Blackwell KT, Plenz D (2006) A comparative voltage and current-clamp analysis of feedback and feedforward synaptic transmission in the striatal microcircuit in vitro. J Neurophysiol 95:737-752.

Hefft S, Jonas P (2005) Asynchronous GABA release generates long-lasting inhibition at a hippocampal interneuron-principal neuron synapse. Nat Neurosci 8:1319-1328.

Holmgren C, Harkany T, Svennenfors B, Zilberter Y (2003) Pyramidal cell communication within local networks in layer $2 / 3$ of rat neocortex. J Physiol 551:139-153.

Hull C, Adesnik H, Scanziani M (2009) Neocortical disynaptic inhibition requires somatodendritic integration in interneurons. J Neurosci 29:8991-8995.

Kawaguchi Y (1995) Physiological subgroups of nonpyramidal cells with specific morphological characteristics in layer II/III of rat frontal cortex. J Neurosci 15:2638-2655.

Kawaguchi Y, Wilson CJ, Augood SJ, Emson PC (1995) Striatal interneurones: chemical, physiological and morphological characterization. Trends Neurosci 18:527-535.

Kita H, Kosaka T, Heizmann CW (1990) Parvalbumin-immunoreactive neurons in the rat neostriatum: a light and electron microscopic study. Brain Res 536:1-15.

Koós T, Tepper JM (1999) Inhibitory control of neostriatal projection neurons by GABAergic interneurons. Nat Neurosci 2:467-472.

Koós T, Tepper JM, Wilson CJ (2004) Comparison of IPSCs evoked by spiny and fast-spiking neurons in the neostriatum. J Neurosci 24:7916-7922.

Le Bé JV, Silberberg G, Wang Y, Markram H (2007) Morphological, electrophysiological, and synaptic properties of corticocallosal pyramidal cells in the neonatal rat neocortex. Cereb Cortex 17:2204-2213.

Mallet N, Le Moine C, Charpier S, Gonon F (2005) Feedforward inhibition of projection neurons by fast-spiking GABA interneurons in the rat striatum in vivo. J Neurosci 25:3857-3869.

Markram H, Lübke J, Frotscher M, Roth A, Sakmann B (1997) Physiology and anatomy of synaptic connections between thick tufted pyramidal neurones in the developing rat neocortex. J Physiol 500:409-440.

Markram H, Wang Y, Tsodyks M (1998) Differential signaling via the same axon of neocortical pyramidal neurons. Proc Natl Acad Sci U S A 95:5323-5328.

McHaffie JG, Stanford TR, Stein BE, Coizet V, Redgrave P (2005) Subcortical loops through the basal ganglia. Trends Neurosci 28:401-407.

Planert H, Grillner S, Robertson B, Silberberg G (2008) Intrinsic properties and synaptic connectivity of different subtypes of the striatal medium spiny neuron. Soc Neurosci Abstr 34:670.8.

Plenz D (2003) When inhibition goes incognito: feedback interaction between spiny projection neurons in striatal function. Trends Neurosci 26:436-443.

Rav-Acha M, Sagiv N, Segev I, Bergman H, Yarom Y (2005) Dynamic and spatial features of the inhibitory pallidal GABAergic synapses. Neuroscience 135:791-802. 
Reyes A, Lujan R, Rozov A, Burnashev N, Somogyi P, Sakmann B (1998) Target-cell-specific facilitation and depression in neocortical circuits. Nat Neurosci 1:279-285.

Somogyi P, Bolam JP, Smith AD (1981) Monosynaptic cortical input and local axon collaterals of identified striatonigral neurons. A light and electron microscopic study using the Golgi-peroxidase transport-degeneration procedure. J Comp Neurol 195:567-584.

Surmeier DJ, Ding J, Day M, Wang Z, Shen W (2007) D1 and D2 dopaminereceptor modulation of striatal glutamatergic signaling in striatal medium spiny neurons. Trends Neurosci 30:228-235.

Taverna S, Canciani B, Pennartz CM (2007) Membrane properties and synaptic connectivity of fast-spiking interneurons in rat ventral striatum. Brain Res 1152:49-56.

Taverna S, Ilijic E, Surmeier DJ (2008) Recurrent collateral connections of striatal medium spiny neurons are disrupted in models of Parkinson's disease. J Neurosci 28:5504-5512.

Tecuapetla F, Carrillo-Reid L, Bargas J, Galarraga E (2007) Dopaminergic modulation of short-term synaptic plasticity at striatal inhibitory synapses. Proc Natl Acad Sci U S A 104:10258-10263.

Tecuapetla F, Koós T, Tepper JM, Kabbani N, Yeckel MF (2009) Differential dopaminergic modulation of neostriatal synaptic connections of striatopallidal axon collaterals. J Neurosci 29:8977-8990.

Tepper JM, Wilson CJ, Koós T (2008) Feedforward and feedback inhibition in neostriatal GABAergic spiny neurons. Brain Res Rev 58:272-281.
Thomson AM, Deuchars J, West DC (1993) Single axon excitatory postsynaptic potentials in neocortical interneurons exhibit pronounced paired pulse facilitation. Neuroscience 54:347-360.

Tsodyks M, Pawelzik K, Markram H (1998) Neural networks with dynamic synapses. Neural Comput 10:821-835.

Tunstall MJ, Oorschot DE, Kean A, Wickens JR (2002) Inhibitory interactions between spiny projection neurons in the rat striatum. J Neurophysiol 88:1263-1269.

Venance L, Glowinski J, Giaume C (2004) Electrical and chemical transmission between striatal GABAergic output neurones in rat brain slices. J Physiol 559:215-230.

Wang Y, Markram H, Goodman PH, Berger TK, Ma J, Goldman-Rakic PS (2006a) Heterogeneity in the pyramidal network of the medial prefrontal cortex. Nat Neurosci 9:534-542.

Wang Z, Kai L, Day M, Ronesi J, Yin HH, Ding J, Tkatch T, Lovinger DM, Surmeier DJ (2006b) Dopaminergic control of corticostriatal long-term synaptic depression in medium spiny neurons is mediated by cholinergic interneurons. Neuron 50:443-452.

Wilson CJ (2007) GABAergic inhibition in the neostriatum. Prog Brain Res 160:91-110.

Wilson CJ, Groves PM (1980) Fine structure and synaptic connections of the common spiny neuron of the rat neostriatum: a study employing intracellular inject of horseradish peroxidase. J Comp Neurol 194:599615. 